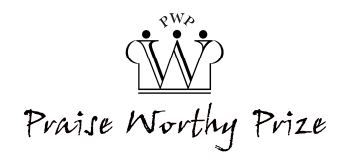

\title{
Prognostics of Onboard Electromechanical Actuators: a New Approach Based on Spectral Analysis Techniques
}

\author{
D. Belmonte, M. D. L. Dalla Vedova, P. Maggiore
}

\begin{abstract}
In the last years, the layout of servomechanisms used in the aeronautical field to actuate the flight controls has changed radically and, nowadays electromechanical actuators (EMAs) are increasingly replacing the older hydraulic powered actuator types. The definition of special monitoring procedures, based on the analysis of the system response and aiming to evaluate the evolution of faults, represents an important task of the modern system engineering taking into account that onboard actuators are typically safety critical items. The present paper proposes a new prognostic procedure centered on the characterization of the state of health of an EMA used in aircraft primary flight controls. This approach, based on the innovative use of a model-based fault detection and identification method (FDI), identifies the actuator actual state of wear of the actuator analyzing proper system operational parameters, able to put in evidence the corresponding degradation path, by means of a numerical algorithm based on spectral analysis techniques. The proposed FDI algorithm has been tested in case of EMA affected by two progressive failures (rotor static eccentricity and stator phase turn-to-turn short-circuit), showing an adequate robustness and a suitable ability to early identify EMA malfunctions with low risk of false alarms or missed failures. Copyright $(2018$ The Authors.

Published by Praise Worthy Prize S.r.l.. This article is open access published under the CC BY-NC-ND license (http://creativecommons.org/licenses/by-nc-nd/3.0/).
\end{abstract}

Keywords: BLDC Motor, Electromechanical Actuator, Prognostics, Spectral Analysis

\section{Nomenclature}

ACE

$\mathrm{ADC}$

BLDC

CEMF

Com

CP

DC

EM

EMA

FDI

FFT

$g_{0}$

$g\left(\vartheta_{r}\right)$

$I_{i}$

$I_{i R M S}$

$I_{\text {ref }}$

$k e_{i}$

$K e_{i}$

$\mathrm{N}_{\mathrm{i}}$

PID

PDE

PHM

RMS

RSE, $\zeta$

RUL

$\mathrm{SC}$

Brushless DC (motor)

Position command

Crossing point

Direct current

Electromagnetic

Electromechanical actuator

Fast fourier transform

Reference current

Power drive electronics

Root mean square

Remaining useful life

Short circuit - short coil
Actuator control electronics

Analog-to-Digital Converter

Counter-electromotive forces

Fault Detection and Identification

Nominal air gap (BLDC motor)

Variable radial air gap (BLDC motor)

Actual current of i-th stator phase $(i=a, b, c)$

RMS value of $i$-th phase current

I-th CEMF constant (one for each branch)

I-th motor trapezoidal modulation function

$\%$ of not short-circuited turns of the i-th coil

Proportional-integral-derivative

Prognositic and Health Management

Rotor Static Eccentricity $\left(\zeta=x_{0} / g_{0}\right)$ $\vartheta_{r} \quad$ Rotor angular position

$x_{0} \quad$ Rotor misalignment

\section{Introduction}

In the last years electromechanical actuators (EMAs) have gradually replaced the actuators based on the hydraulic power in flight command actuation systems. EMAs are driven by electric motors that transfer rotational power to the control surfaces by means of gears. Compared to electrohydraulic systems, EMAs offer several advantages: in particular, overall system weight is reduced, maintenance is simplified and hydraulic fluids, which are often leaking, flammable or polluting, can be completely eliminated [1]. Since most of these actuators are safety critical, scheduled maintenance tasks and design redundancy are currently applied in order to guarantee that the system always operates in safe conditions. However, unexpected and extreme operative scenarios may lead to damage them and unscheduled maintenance becomes necessary, increasing risks and costs and generating possible impact on the mission. In this regard, according to [2], the reliability of modern systems is even more based on the proper support of diagnostics and prognostics. Both represent one of the most effective method to mitigate life cycle costs without losing reliability and guarantee, in compliance with regulations, the basis for an effective 
health management of integrated components, subsystems and systems [3]. The practice of monitoring and analyzing the system response, through electrical signal acquisition, to evaluate the fault is the goal of the Prognositic and Health Management (PHM) [4].

In general, as reported in [5], the prognostics purpose is to perform an early Fault Detection and Identification (FDI) and, then, to provide real-time data on the current status of the system and to calculate the Remaining Useful Life (RUL) before a fault occurs or a component becomes unable to perform its functions at a given level.

In aerospace disciplines, prognostic strategies often apply to onboard equipment (typically non-linear mechatronic systems) and, by the monitoring of some functional parameters of the component involved, they have to predict progressive failures at an early stage and to determine the source of irregular behaviors.

Literature proposes several FDI strategies, often conceived and implemented to specific problems or welldefined technical fields [6]; as regards onboard actuation systems based upon EMAs, it is possible to mention the followingmain FDI techniques:

- model-based techniques founded on the comparison between the real system and related monitoring model (e.g. deterministic methods based upon appropriate merit coefficients [7]-[8], genetic algorithms [9]-[10] or further probabilistic approaches such as the simulated annealing method [11]);

- approaches based on the spectral analysis of welldefined signals (i.e. related to peculiar behaviors of the system that allow a timely identification of these incipient failures) and, generally, performed by Fast Fourier Transforms (FFT) methods [12]-[13];

- hybrid approaches that exploit a suitable combination of the above methods in order to identify the health of the system [14];

- identification and classification algorithms based on artificial neural networks [15]-[17].

The concepts reported in this paper are related to the design of a reliable and fast FDI routine focused on the diagnosis model-based approach and, in particular, on the parametric estimation task. It must be noted that PHM approaches can be applied on EMAs in a more efficient way (if compared to the case of the most known electrohydraulic actuators), because in EMAs additional sensors are not required. Indeed, prognostic strategies normally involve the monitoring of parameters that in EMAs are already provided by the same sensors of the system monitor and control functions.

This study is focused on the development a prognostic technique able to identify failure precursors alerting that the consideredEMAis degrading (exhibiting anomalous behaviors). In particular, in this paper two progressive failures are considered: partial stator phase turn-to-turn Short Circuit (SC) and Rotor Static Eccentricity (RSE).

The obtained results prove that this FDI method is suitably robust and it is also able to provide early and reliable identifications of any system malfunctions, reducing the risk of false alarms or undetected failures.
Furthermore, it is able to operate on the base of data collected by sensors that already equip the actuation system or derived from the post-processing of the real raw measurements (virtual sensors).

The authors adopted this strategy because, as it is known, the introduction of additional components in the EMA transducers network could generate the onset of integration problems such (e.g. redesign of the hardware layout, software modifications, compatibility problems, redundancies, reduction of overall reliability and increase in costs) [7] and, at the same time, it would make much more difficult the implementation of the aforementioned prognostic method on preexisting equipment. In addition, it must be noted that this integration between real and virtual sensors, combining and processing information from multiple sources, can increase the effectiveness and robustness of the FDI algorithm. From an operational point of view, this FDI approach can be easily integrated into a system control process periodically performed by maintenance personnel (e.g. in pre-flight checks).

\section{EMA Reference Model}

The actuation system considered by the authors is an electromechanical position servomechanism typically used in primary and secondary flight controls (Fig. 1).

As suggested by [18], the aforesaid system can be studied subdividing it into six principal subsystems:

- a control electronics unit (defined as ACE in Fig. 1) that closes the position feedback loop- comparing the command input with the actual position, elaborating corrective actions (by means of a PID control logic [19]-[20]), and regulating the reference current $\left(I_{\text {ref }}\right)$;

- a Power Drive Electronics unit (PDE) regulating the three-phase electrical power;

- a BLDC three-phase electrical motor;

- a gearbox that adapts the motor outputs reducing its rotor angular speed (and, at the same time, increasing the supplied mechanical torque);

- a nut-screw system that converts motor rotation into linear motion (ball screws or roller screws are normally used because, with respect to acme screws, they provide lower frictions and higher efficiencies);

- a network of sensors (SENSORS) able to close the feedback chains (i.e. control rings acting on phase currents, motor angular speed and output position).

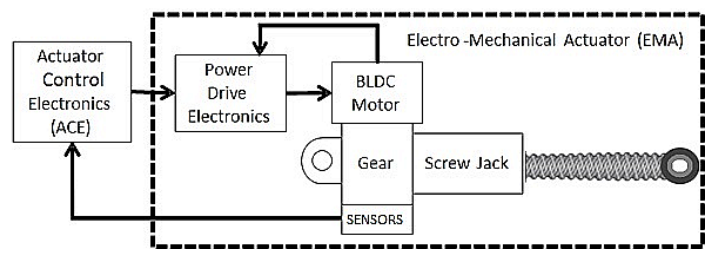

Fig. 1. Electromechanical actuator (EMA) scheme

In this work a Matlab-Simulink numerical model is used, in place of a physical test bench, to provide reference data to be analyzed by the prognostic FDI 
algorithm. It must be noted that this model is very detailed, in particular regarding the electromagnetic interaction between the BLDC rotor and stator.

This EMA numerical model is widely described in [11] and it is consistent with the block diagram of Fig. 2.

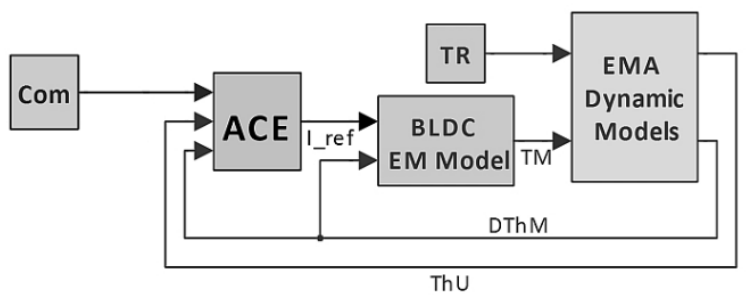

Fig. 2. Proposed EMA Matlab-Simulink block diagram

It consists of the following five blocks:

- an input block (named as Com in Fig. 2) generating the various position commands;

- an ACE block that simulates the real EMA actuator control electronics, closing its feedback loops and calculating the reference current $I_{\text {ref }}$ [21];

- a block that simulates the dynamic response of the electric motor (BLDC EM Model) implementing the electromagnetic model of the trapezoidal BLDC motor and the characteristics of the power control electronics: this block calculates the mechanical torque developed by the motor as a function of the voltage regulated by the three-phase inverter [14];

- a block simulating the EMA mechanical behavior by a 2 d.o.f. dynamic system (EMA Dynamic Models);

- another input block (named as TR in Fig. 2) which allows simulating the aerodynamic forces (if any) acting on the control surface driven by the EMA.

This numerical model simulates the behavior of the real EMA taking also into account the effects of BLDC motor degradation and non-linearities such as partial phase short circuit a rotor eccentricity [22]-[25], end-oftravels, compliance and backlashes acting on gearbox and ballscrew [26]-[28], ADC conversion of the feedback signals, electrical noise acting on the signal lines and electrical offset of the position transducers [7] and dry friction acting on bearings, gears, hinges and screws [29].

\section{EMA Failures Modelling}

Since the use of electromechanical actuators in the aeronautical field is quite recent, the statistics about their failure modes and the related effects are still not very extensive and detailed [30]. EMAs can be subjected to a wide number of possible failure modes, involving both hardware and software items. In general, their progressive failures can be classified into four main areas: electronics (e.g. ACE and sensors) [27]-[28], electric motor [11], [31], mechanical (e.g. gearboxes and screw actuators) or structural failures [31]-[32]. As regards the main failure modes affecting BLCD motors, as reported in [13], they are generally due to progressive turn-to-turn type short circuits on the stator coils and rotor static eccentricity (e.g. due to bearing wears). Short Circuit (SC) is usually due to thermal effects (e.g. motor overheating or stator current peaks) which locally compromise the insulation of the coil windings.

This type of failure usually starts between some adjacent turns of the same phase (turn-to-turn failure), then it grows until propagates to the entire coil (progressively increasing the failure magnitude). In fact, at the ends of the short-circuited coil the commanded voltage remains almost the same, but, as the damage grows, its overall resistance diminishes; this determines the increase of the phase current (i.e. the current circulating in the winding) and, therefore, causes a localized heating in the conductor which, gradually, amplifies its propagation.

The Rotor Static Eccentricity (RSE) of an electric motor $\zeta$ is defined as the ratio between the rotor misalignment $x_{0}$ and the nominal radial air gap $\mathrm{g}_{0}$; as shown in Fig. 3, the said misalignment equals to the distance between the rotor axis and the axis of symmetry of the stator.

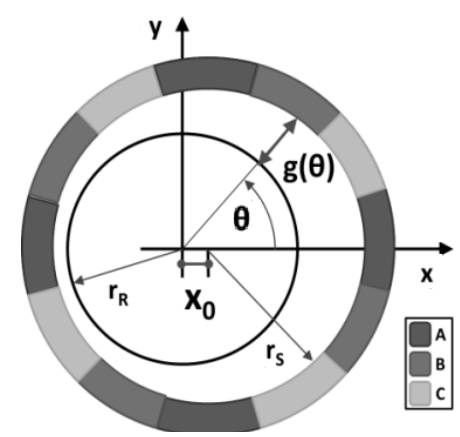

Fig. 3. Schematic of the BLDC Motor geometrical reference system

As shown in Fig. 3, the local value of the radial gap $g$ $\left(\vartheta_{r}\right)$, in case of RSE, varies during the motor rotation as reported in (1). For motors having two or more polar couples, this fault generates a periodic modulation of the magnetic flux depending on the rotor position $\vartheta_{r}$ :

$$
g\left(\vartheta_{r}\right)=g_{0}\left(1+\zeta \cos \left(\vartheta_{r}\right)\right)
$$

where $\zeta=\frac{x_{0}}{g_{0}}$.

The effects of these failures on the electrical features of the BLDC motor (e.g. winding resistance, windings inductance and counter-electromotive forces) have been modelled through the simplified numerical model proposed in [33]. In particular, their effects on the magnetic coupling between stator and rotor have been simulated by varying values and angular modulation of counter-electromotive forces (CEMF) coefficients; this method acts on the three CEMF constants $C e_{i}$ (one for each motor branch) implemented in the BLDC EM Model block (Fig. 1), by modulating their trapezoidal modulation functions $K e_{i}$ as a function of coil SC 
percentage, RSE $\zeta$ and angular position $\vartheta_{r}$ :

$$
k e_{i}=K e_{i} \cdot C e_{i} \cdot\left(1+\zeta \cos \left(\vartheta_{r}\right)\right) \quad-\quad i=a, b, c
$$

As proposed in [34], the three constants $k e_{a}, k e_{b}, k e_{c}$ obtained from (2) are used to calculate the counterelectromotive forces induced on the stator windings and, therefore, to evaluate the value of the mechanical torque contributions generated by the three motor phases.

Indeed, an early identification of these failure precursors allows adopting countermeasures able to limit an increasingly faster propagation of failures on sensors and electrical components [35]-[36]. It should be noted that the BLDC EM subsystem of the proposed EMA numerical model (shown in sections II and III) is implemented in the Simscape Power Systems ${ }^{\mathrm{TM}}$ multidomain simulation environment; therefore, unlike some algorithms available in the literature (often based upon a linear modelling of the EM phenomena), it is able to calculate the instantaneous value of each phase current $\left(I_{a}, I_{b}, I_{c}\right)$ even in the case of unbalanced stator EM circuit (such as, for instance, in case of partial coil short circuit or rotor static eccentricity).

Therefore, SC and RSE failures are related to the corresponding dynamic response of stator phase currents (that will be adopted as failure precursors) by means of an algorithm based on the Fourier spectral analysis of these three signals; in order to attenuate high-frequency noises and disturbances affecting the said signals, each phase current is previously filtered by a third order lowpass filter [37].

\section{EMA Spectral Algorithm and Operating Map}

The behavior of the simulated EMA actuation system has been evaluated analyzing its dynamic response for a very high step position command in order to quickly saturate the electromechanical actuator controller and, then, reachingits maximum actuation velocity (without aerodynamic load).

Indeed, this kind of input allows to quickly saturate the EMA control rings generating an open-loop dynamic response [34] (i.e. following this step position command, the actuator starts and, after a short transient time, reaches its maimum no-load velocity).

The whole test simulation time amounts to one second and, for each simulation, all filtered phase currents $\left(I_{a}, I_{b}\right.$, $I_{c}$ ) have been acquired and used as prognostic precursors to evaluate contemporary the corresponding RSE and the percentage of SC relative to each stator phases.

The Root Mean Square (RMS) of a given signal time history represents a measure of its overall energy and it is often used to extract signal features and trending data for FDI fault detection and identification processes. To avoid numerical problems of aliasing, the time history of the signal must be digitized by a proper sample rate of $10^{-6}$ samples/s (for a total of $10^{+6}$ samples); then, the RMS value can be estimated as shown in (3):

$$
R M S=\sqrt{\frac{1}{N} \sum_{i=1}^{N} X^{2}(i)}
$$

The RMS algorithm is applied for each filtered phase current $\left(I_{a}, I_{b}, I_{c}\right)$ as a function of the percentage of turns of the stator coils still not short-circuited $\left(N_{i}\right.$, where $i=a$, $b, c$ refers to the different stator phases of the three-phase BLDC motor); operatively speaking, the results reported in this paragraph are calculated as a function of a progressive SC acting on the coil of the phase " $a$ ", with $N_{a}$ varying from $75 \%$ to $100 \%$ with a $1 \%$ increasing step.

Three signals called $I_{a R M S}, I_{b R M S}$, and $I_{c R M S}$ are calculated: Fig. 4 shows their evolutions as a function of $N_{a}$ in case of RSE $=0$.

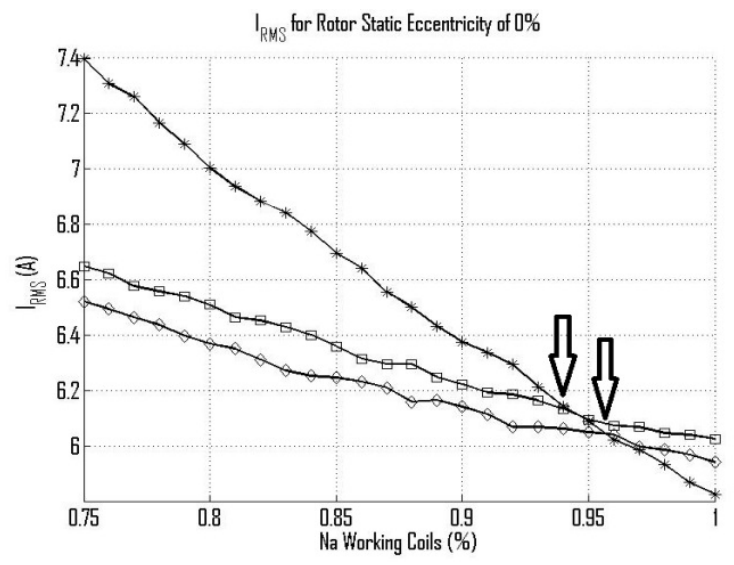

Fig. 4. Evolution of the three RMS phase currents as a function of $\mathrm{N}_{\text {a }}$ (working coils percentage) for RSE $=0 \%$. The black arrows highlight the CPs respectively between $I_{a R M S}$ (asterisk) - $I_{b R M S}$ (squares), and $I_{a R M A}$ (asterisk) $I_{c R M S}$ (diamonds)

The progressive SC degradation of the single phase " $a$ " induces an increasing of the three phases current RMS as a function of $N_{a}$ percentage.

Moreover, the stator phase damaged by progressive SC degradation puts in evidence an growing slope of its phase current, higher than the other ones, that increases proportionally with the said failure (Fig. 4).

The $I_{a}$ RMS, identifies two critical points, called Crossing Points (CPs), in which it crosses the other currents functions $I_{b} R M S$ and $I_{c} R M S$ (relating to the initial operating condition with $N_{b}=N_{c}=1$ ).

The SC degradation, acting on phase " $a$ " unbalances the stator electrical circuit influencing the other alimentation phases, although these are operating in nominal conditions without SC, and modifying the corresponding RMS values of the filtered phase currents.

Important information is given by the evaluation of the CPs related to short coil degradation percentage on evaluating RMS of filtered phase currents calculated by the authors' EMA simulation model.

Therefore, the proposed methodology can evaluate with suitable accuracy the working coils percentage $N_{a}$ 
for a single damaged phase by using the aforesaid failure map, by considering the CPs as results of a real-time measurement, performed by the EMA numerical model under the same operational conditions.

The model-based test bench, considers a specific EMA model and the related technical parameters as simulation input, but it is possible to calculate other specific failure maps to evaluate short coils degradation by changing the input technical parameters. The failure map showed in Fig. 4 considers a fixed rotor static eccentricity equal to zero, but this approach is too simplified to give an exhaustive description of the interaction between short coil degradation phenomena and rotor static eccentricity. The rotor static eccentricity has a strong influence on the proposed method, because both the type of considered failures cause different effects on the chosen prognostic precursors in the described method.

Indeed, the rotor static eccentricity due to mechanical wear reduces clearance between rotor and stator surfaces as shown in Fig. 3, so it is possible to associate to minimum clearance a minimum magnetic resistance to the minimum clearance. The RMS filtered phase current values decrease related to the minimum clearance at the same voltage; in the proposed test case, the supply phase " $a$ " is at the same time interested by the short coil degradation and minimum clearance associated with rotor static eccentricity. In other terms, increasing rotor static eccentricity causes opposed effects related to short coil induced effects where the RMS filtered phase current increases, for the damaged phase, strongly with increasing coils degradation. Increasing RSE modifies the overall behavior of the failure map and, in particular, the position (in terms of $N_{a}$ and RMS current) of related CPs. In case of RSE equal to $50 \%$, the percentage of SC damage $\left(N_{a}\right)$ associated with the corresponding CPs (Fig. 5) results higher compared to the case of rotor static eccentricity equal to zero (Fig. 4).

It should be noted that each of the aforesaid CPs maps is calculated considering only two of the three filtered phase currents, as shown respectively in Fig. 6 (referred to $I_{a R M S}$ and $I_{b R M S}$ ) and in Fig. 7 (for $I_{a R M S}$ and $I_{c R M S}$ ).

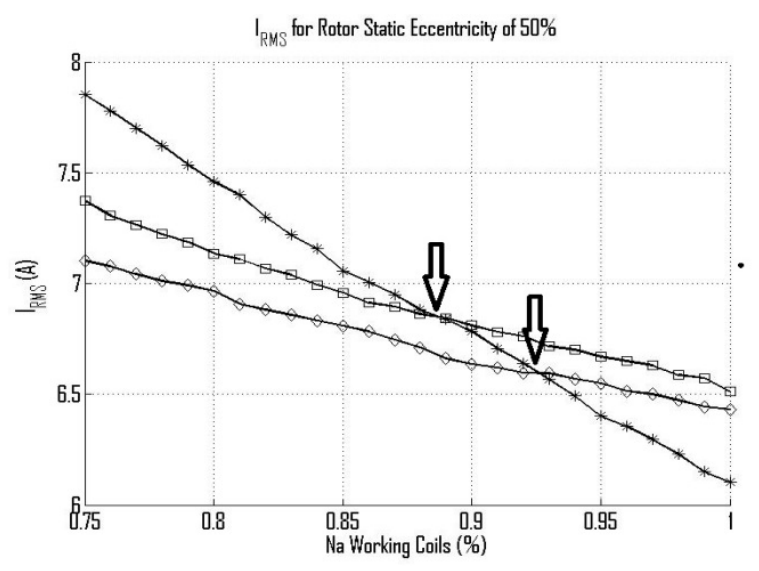

Fig. 5. Evolution of the three RMS phase currents as a function of $\mathrm{N}_{\mathrm{a}}$ (working coils percentage) for RSE $=50 \%$. The black arrows highlight the CPs respectively between $I_{a R M S}$ (asterisk) - $I_{b R M S}$ (squares), and $I_{a R M A}$ (asterisk) $I_{c R M S}$ (diamonds)

These failure maps could give an evaluation of the rotor static eccentricity, even though crossing points data presents scatter distribution as a function of $N_{a}$ working coils percentage. Scattered data could be interpolated by a least squares fitting interpolation function of third grade, to define a reference function able to identify given measured cross points, between two filtered phase currents, both the short coils degradation and an evaluation of a rotor static eccentricity.

The reference interpolation function for $I_{a R M S}, I_{b R M S}$ CPs is indicated in (4), whereas the interpolation function for $I_{a R M S}, I_{c R M S} \mathrm{CPS}$ is indicated in (5):

$$
\begin{gathered}
184 X^{3}-396 X^{2}+231 X-23=0 \\
-4984 X^{3}+14207 X^{2}-13511 X+4293=0
\end{gathered}
$$

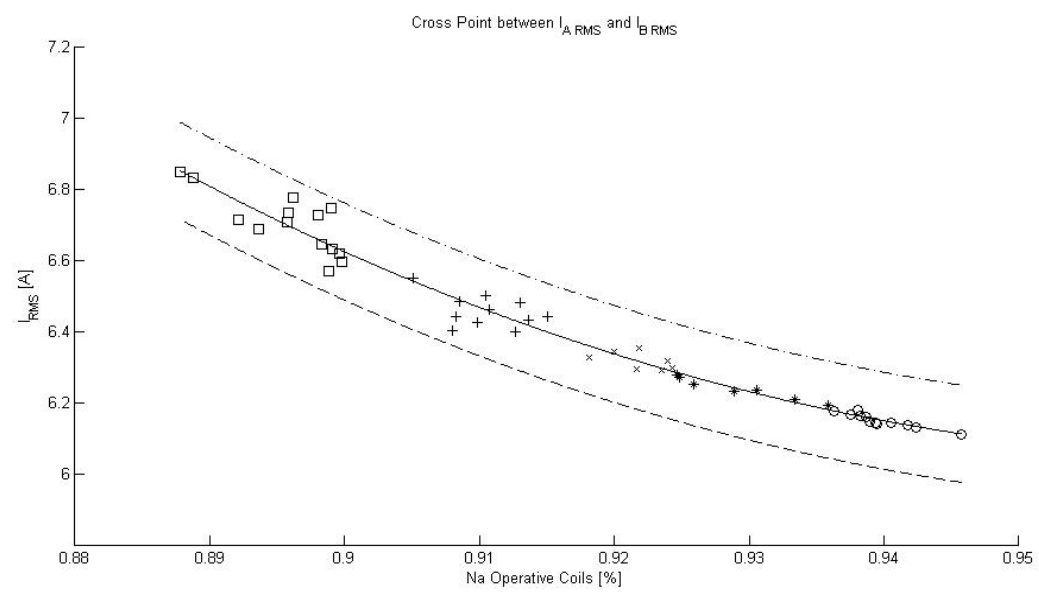

Fig. 6. Evolution of Crossing Points between $I_{a R M S}$ and $I_{b R M S}$ as a function of RSE from $0 \%$ to $50 \%$ and progressive short coil degradation. The Crossing Points are classified as rotor static eccentricity intervals: from $0 \%$ to $12 \%$ (circle), from $13 \%$ to $19 \%$ (asterisk), from $20 \%$ to $27 \%$ (cross), from $28 \%$ to $38 \%$ (plus), from $39 \%$ to $50 \%$ (square) 


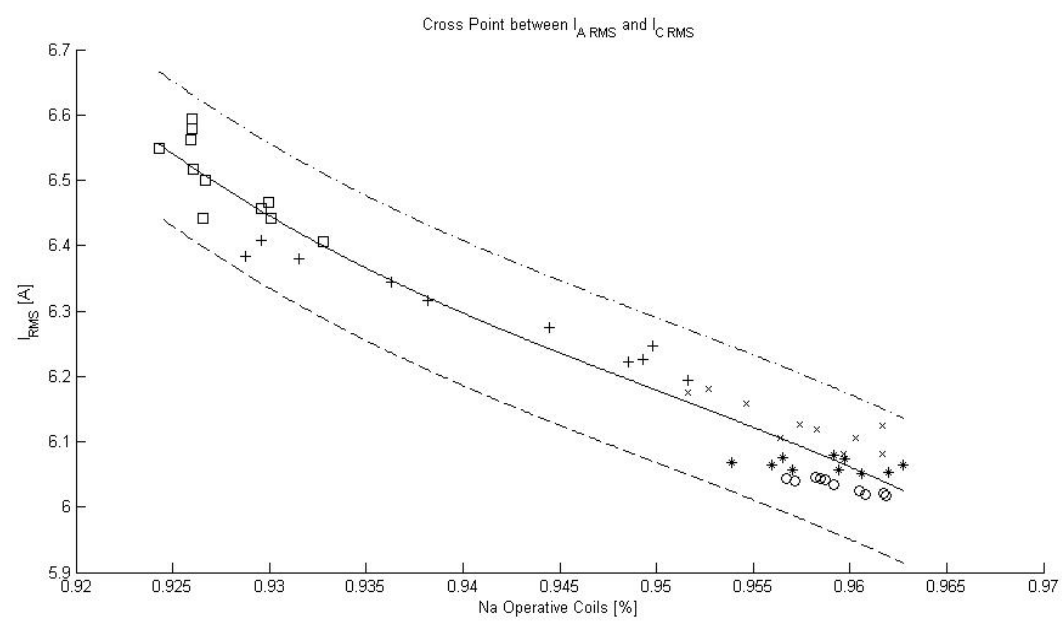

Fig. 7. Evolution of Crossing Points between $I_{a R M S}$ and $I_{c R M S}$ as a function of RSE from $0 \%$ to $50 \%$ and progressive short coil degradation. The Crossing Points are classified as rotor static eccentricity intervals: from $0 \%$ to $12 \%$ (circle), from $13 \%$ to $19 \%$ (asterisk), from $20 \%$ to $27 \%$ (cross), from $28 \%$ to $38 \%$ (plus), from $39 \%$ to $50 \%$ (square)

The proposed FMs consider a translation of the reference function to define upper and lower limits translating the reference curve to the maximum distance between scattering data and reference function added with a 30\% margin. Therefore, the upper and lower translating functions define on diagram an evaluation 2D areas able to associate an interval of Short coil degradations with rotor static eccentricity evaluation for crossing points measurement, on real EMA under the simulated boundary conditions interval.

In other words, the $\mathrm{CP}$ for filtered phase currents, in simulated failure conditions for both SC and RSE degradations, can define combined failure maps able to estimate the concurrent failures by identifying evaluation areas based on least squares fitting curves and related upper and lower translations. The failure map shown in Fig. 6, that is related to CPs between $I_{a}$ RMS and $I_{b}$ RMS , represents a more effective FDI tool than the other maps (based on CPs between $I_{a \text { RMS }}$ and $I_{c \text { RMS }}$ and shown in Fig. 7), because it identifies well-defined RSE growing intervals, avoiding areas superposition that could reduce its accuracy. If the RSE percentage is higher than 30\%, it could be necessary to select a higher alarm threshold (corresponding to the maximum deviation of the CPs with respect to the RMS current interpolating function); this value can be identified as a function of the CPs dispersion on the corresponding failure maps (Fig. 6 and Fig. 7). The RSE magnitude considered for prognostics is typically smaller than the $50 \%$ and its FDI upper limits is often evaluated around 25-30\%; indeed, bigger RSE percentages could produce macroscopic failure effects on EMA behaviors (e.g. degrading its performances beyond acceptable limits and compromising its effectiveness).

It should be noted that the evolution of these two progressive failure types (RSE and stator SC) presents two different time scales; in details, the RSE is mainly due to mechanical wear phenomena and, therefore, it is correlated to a growth dynamics typically much slower than the SC failures affecting the BLDC motor.

In conclusion, this method can evaluate different fault conditions, performing the FDI analysis also in case of combined failures (e.g. RSE and stator SC). It should be noted that this fact is not in itself obvious because these failures generate contrasting effects on the adopted prognostic parameters (i.e. stator phase currents) that, sometimes, they could hide one each other. Other modelbased FDI methods presented in literature, implementing FEM analysis and/or statistical methodology, are interactively computed up to the convergence of suitable fitness functions (e.g. L1-norm or L2-norm error), in order to evaluate the EMA health status [38]. Therefore, the proposed method performs an early FDI analysis by calculating, in off-line mode, the aforesaid failure maps (based on an EMA high fidelity simulation model) and, then, by comparing the so obtained results with the corresponding prognostic variables provided by the real monitored EMA during a real-time experimental acquisition. At the operational level, especially if compared with FDI techniques based on high fidelity models, the proposed method (based upon off-line calculated failure maps and low fidelity approaches) requires reduced time of calculation and smaller computational resources and so, allowing periodical FDI checks (e.g. real-time or almost real-time controls during the flight or scheduled prognostic tests during the usual on-field maintenance procedures), it increases the safety of the whole system (i.e. the aircraft).

\section{Conclusion}

The proposed method allows to calculate specific operating maps for many different EMA models: it is possible to adjust the proposed numerical model to a particular type of EMA by setting some technical parameters and then define the corresponding operational map (in order to evaluate the coils degradation taking into account rotor static eccentricity effects).

The EMA failure precursors (provided by the onboard monitoring system) are compared with the related operating map in order to evaluate, during a preflight 
test, the percentage of coil short-circuit affecting a single alimentation phase, avoiding degraded flight command performances and so improving the safety margin of the whole actuation system. These operational maps are then integrated with the above said filtered RMS phase current module in order to guarantee an accurate evaluation of the health state of the real EMA (performed during maintenance operations and pre-flight tests, as indicated in previous paragraphs). The obtained results encourage the extension of the proposed technique to more challenging occurrences such as the interactions between electrical (e.g. SCs affecting the stator coils) and mechanical failures due to wear (e.g. RSE, dry friction and backlashes acting on mechanical transmission); in fact, these faults are frequently difficult to evaluate with suitable accuracy because they are usually characterized by very different time scales and sometimes produces opposite effects on prognostic precursor analysis.

In order to achieve these goals, the proposed actuator model should be further developed, implementing new mathematical model representative of the considered phenomena and modeling in detail some critical components, so as to extending spectral analysis and investigating in a deeper way interactions due to these combined failures.

\section{Acknowledgements}

The authors wish to extend a heartfelt thanks to Prof. Lorenzo Borello, Ing. Giovanni Oddone and Mr. Mario Plancher, for their essential support in the ideation and and development of these research activities.

\section{References}

[1] J. Marè, Electro-mechanical Actuators, in Aerospace Actuators 2: Signal-by-Wire and Power-by-Wire (Wiley, New York, 2017).

[2] G. Vachtsevanos, F. Lewis, M. Roemer, A. Hess, B. Wu, Intelligent fault diagnosis and prognosis for engineering systems (Wiley, New York, 2006).

[3] K. Goebel et al., Prognostics: the science of making prediction (CreateSpace Independent Publishing Platform, 2017).

[4] C. S. Byington, W. Watson, D. Edwards, P. Stoelting, A modelbased approach to prognostics and health management for flight control actuators, IEEE Aerospace Conference Proc. (2014).

[5] W. F. Bonnice, W. Baker, Intelligent fault diagnosis and failure management of flight control actuation systems, NASA-CR177481 (1988).

[6] T. Sutharssan, S. Stoyanov, C. Bailey, C. Yin, Prognostic and health management for engineering systems: a review of the datadriven approach and algorithms, The Journal of Engineering 2015(7) (2015), 215-222.

[7] L. Borello, M.D.L. Dalla Vedova, G. Jacazio, M. Sorli, A prognostic model for electrohydraulic servovalves, Proc. of the Annual Conference of the Prognostics and Health Management Society (2009).

[8] Adouni, A., Ben Hamed, M., Sbita, L., Online Steady State Detection and Isolation of Fault Measurements Occurred by Sensors and Actuator, (2014) International Review on Modelling and Simulations (IREMOS), 7 (5), pp. 768-774. doi:https://doi.org/10.15866/iremos.v7i5.4718

[9] M. Alamyal, S. M. Gadoue, B. Zahawi, Detection of induction machine winding faults using genetic algorithm, Diagnostics for Electric Machines, Power Electronics and Drives 9th IEEE Int. Symposium, 157-161 (2013).
[10] A. Raie, V. Rashtchi, Using a genetic algorithm for detection and magnitude determination of turn faults in an induction motor, Electrical Engineering 84(5) (2002), 275-279.

[11] M. D. L. Dalla Vedova, A. Germanà, P. Maggiore, Proposal of a new simulated annealing model-based fault identification technique applied to flight control EM actuators, Risk, Reliability and Safety: Innovating Theory and Practice - Proceedings of the 26th European Safety and Reliability Conference, ESREL 2016, 313-321 (2016)

[12] P. Maggiore, M. D. L. Dalla Vedova, L. Pace, Proposal of prognostic parametric method applied to an electrohydraulic servomechanism affected by multiple failures, WSEAS Transactions on Environment and Development (2014), 478-490.

[13] D. Belmonte, M. D. L. Dalla Vedova, P. Maggiore, Electromechanical servomechanisms affected by motor static eccentricity: proposal of fault evaluation algorithm based on spectral analysis techniques, Safety and Reliability of Complex Engineered Systems - Proceedings of the 25th European Safety and Reliability Conference, ESREL 2015, 2365-2372 (2015).

[14] M. D. L. Dalla Vedova, P. Maggiore, L. Pace, A. Desando, Evaluation of the correlation coefficient as a prognostic indicator for electromechanical servomechanism failures, International Journal of Prognostics and Health Management 6 (2015), 1-13.

[15] M. Battipede, M. D. L. Dalla Vedova, P. Maggiore, S. Romeo, Model based analysis of precursors of electromechanical servomechanisms failures using an artificial neural network, AIAA Modeling and Simulation Technologies Conference, (2015).

[16] M. D. L. Dalla Vedova, D. De Fano, P. Maggiore, Neural network design for incipient failure detection on aircraft EM actuator, International Journal of Mechanics and Control 17(1) (2016), 7783.

[17] Pradeep, J., Devanathan, R., Fault Diagnosis of PMSM Using Artificial Neural Network, (2014) International Review on Modelling and Simulations (IREMOS), 7 (5), pp. 760-767. doi:https://doi.org/10.15866/iremos.v7i5.3156

[18] I. Moir, A. Seabridge, Aircraft Systems: Mechanical, Electrical and Avionics Subsystems Integration (Wiley, New York, 2008).

[19] Gaya, M., Bisu, A., Salim, S., Madugu, I., Yusuf, L., Kaurangini, M., Khairi, M., Optimal PID Design Approaches for an Inverted Pendulum System, (2016) International Review of Automatic Control (IREACO), 9 (3), pp. 167-174. doi:https://doi.org/10.15866/ireaco.v9i3.9160

[20] Hernández Beleño, R., Mora Gonzales, P., Avilés Sánchez, O., Ferreira, J., Dynamic Modeling and PID Control of an Underwater Robot Based on the Hardware-in-the-Loop Method, (2016) International Review of Mechanical Engineering (IREME), 10 (7), pp. 482-490. doi:https://doi.org/10.15866/ireme.v10i7.9037

[21] I. Todić, M. Miloš, M. Pavišić, Position and speed control of electromechanical actuator for aerospace applications, Tehnicki Vjesnik 20(5) (2013), 853-860.

[22] T. A. Haskew, D. E. Schinstock, E. Waldrep, Two-Phase On' Drive Operation in a Permanent Magnet Synchronous Machine Electromechanical Actuator, IEEE Transactions on Energy Conversion 14, (1999).

[23] A. Halvaei Niasar, H. Moghbelli, A. Vahedi, Modelling, Simulation and Implementation of Four-Switch Brushless DC Motor Drive Based On Switching Functions, IEEE EUROCON 2009, (2009).

[24] B. K. Lee, M. Ehsani, Advanced Simulation Model for Brushless DC Motor Drives, Electric Power Components and Systems, 31(9) (2003), 841-868.

[25] M. Çunkas, O. Aydoğdu, Realization of Fuzzy Logic Controlled Brushless DCMotor Drives using Matlab/Simulink, Mathematical and Computational Applications 15(02) (2010), 218-229.

[26] L. Borello, G. Villero, M. D. L. Dalla Vedova, New asymmetry monitoring techniques: effects on attitude control, Aerospace Science and Technology 13(8) (2009), 475-487.

[27] L. Borello, M. D. L. Dalla Vedova, Flaps Failure and Aircraft Controllability: Developments in Asymmetry Monitoring Techniques, Journal of Mechanical Science and Technology (JMST) 28(11) (2014), 4593-4603.

[28] Huikuri, M., Jansen, J., Lomonova, E., Pyrhönen, J., Adaptive Feedforward Control of an Industrial H-Drive Positioning with 
Permanent Magnet Linear Motors, (2015) International Review on Modelling and Simulations (IREMOS), 8 (4), pp. 399-409. doi:https://doi.org/10.15866/iremos.v8i4.6549

[29] L. Borello, M. D. L. Dalla Vedova, A dry friction model and robust computational algorithm for reversible or irreversible motion transmission, International Journal of Mechanics and Control 13(2) (2012), 37-48.

[30] A. De Martin, G. Jacazio, G. Vachtsevanos, Windings fault detection and prognosis in electro-mechanical flight control actuators operating in active-active configuration, International Journal of Prognostics and Health Management 8(2) (2017).

[31] F. L. J Van Der Linden, N. Dreyer, A. Dorkel, EMA Health Monitoring: An Overview. Proc. of Recent Advances in Aerospace Actuation Systems and Components, March 16-18, Toulouse, France, (2016).

[32] C. Lessmeier, O. Enge-Rosenblatt, C. Bayer, D. Zimmes, Data acquisition and signal analysis from measured motor currents for defect detection in electromechanical drive systems. Proc. of the European Conference of the Prognostics and Health Management Society, July 8-10, Nantes, France, (2014).

[33] B. W. Kim, K. T. Kim, J. Hur, Simplified impedance modeling and analysis for inter-turn fault of IPM-type BLDC motor, Journal of Power Electronics 12(1) (2012), 10-18.

[34] D. Belmonte, M. D. L. Dalla Vedova, C. Ferro, P. Maggiore., Electromechanical actuators affected by multiple failures: Prognostic method based on spectral analysis techniques, AIP Conference Proceedings 1836, 020020, AIP Publishing (2017).

[35] A. Ginart, D. Brown, P. Kalgren, M. Roemer, On-line Ringing Characterization as a PHM Technique for Power Drives and Electrical Machinery, Autotestcon (IEEE),(2007).

[36] A. Ginart, D. Brown, P. Kalgren, M. Roemer, Inverter Power Drive Transistor Diagnostic and Extended Operation under OneTransistor Trigger Suppression, Proc. Of the Applied Power Electronics Conference and Exposition, (APEC),(2008).

[37] E. E Ngu, K. Ramar, R. Montano, V. Cooray, Fault characterisation and classification using wavelet and fast Fourier transforms, WSEAS Trans. on Signal Processing 4(7) (2008).

[38] E. Woecker, Methods for Inter Coil Short Circuit Detection in Excitation Winding Coils of Turbo Generator in Operation and in Standstill Condition, Proc. of the CIGRE Colloquium on new development of rotating electrical machines, (2011).

\section{Authors' information}

Department of Mechanical and Aerospace Engineering (DIMEAS), Politecnico di Torino, C.so Duca degli Abruzzi, 24 - Torino, Italy.

E-mails: dario.belmonte@ polito.it matteo.dallavedova@polito.it paolo.maggiore@polito.it

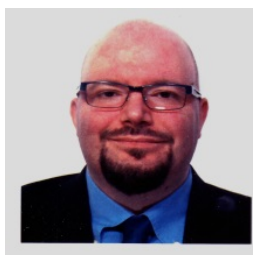

Dario Belmonte received the M.Sc. at Politecnico di Torino in 2011. Since 2011 he worked as assistant researcher at the Department of Mechanics and Aerospace Engineering and GE Avio Aero srl. His research activity is mainly focused on analysis and numerical simulation of dynamic systems and aerospace servomechanism, integrating correlations between FEM and CAT (experimental data) related to structural vibrational analysis for Gear Box and Turbine modules.

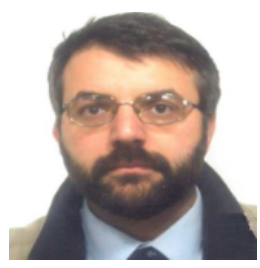

Matteo D. L. Dalla Vedova received the M.Sc. and the Ph.D. from the Politecnico di Torino in 2003 and 2007, respectively. He is currently Assistant Professor in the Department of Mechanics and Aerospace Engineering. His research activity is mainly focused on the aeronautical systems engineering and, in particular, is dedicated to design, analysis and numerical simulation of on board systems, study of secondary flight control systems and conception of related monitoring strategies, development of prognostic algorithms for aerospace servomechanism and study of innovative primary flight control architectures.

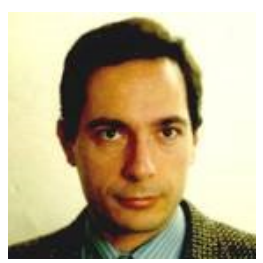

Paolo Maggiore is a professor at the Mechanical and Aerospace Engineering Department of Politecnico di Torino, that joined in 1992, where he teaches aerospace general systems engineering. Currently his students are involved in projects ranging from hydrogen fuel cell powered airplanes and UAVs, and health monitoring of flight controls, to multidisciplinary design optimization of aerospace systems design. His main research topics are: energy management and hydrogen fuel cells power units, Safety and Reliability of Mechatronic Systems, System Engineering and Multi-Disciplinary Design Optimization, Design and Control of UAVs, Prognostic and Health Monitoring. 